\title{
Essential role of the cryptic epitope SLAYGLR within osteopontin in a murine model of rheumatoid arthritis
}

\author{
Nobuchika Yamamoto, ${ }^{1}$ Fumihiko Sakai, ${ }^{1}$ Shigeyuki Kon, ${ }^{2,3}$ Junko Morimoto, ${ }^{2}$ \\ Chiemi Kimura, ${ }^{2}$ Harumi Yamazaki, ${ }^{1}$ Ikuko Okazaki, ${ }^{1}$ Nobuo Seki, ${ }^{1}$ Takashi Fujii, ${ }^{1}$ \\ and Toshimitsu Uede ${ }^{2}$
}

${ }^{1}$ Exploratory Research Laboratories, Fujisawa Pharmaceutical Co., Ibaraki, Japan

${ }^{2}$ Institute for Genetic Medicine, Hokkaido University, Hokkaido, Japan

${ }^{3}$ Immuno-Biological Laboratory, Gunma, Japan

\begin{abstract}
It has been shown that osteopontin (OPN) plays a pivotal role in the pathogenesis of rheumatoid arthritis (RA). However, the molecular mechanism of OPN action is yet to be elucidated. Splenic monocytes obtained from arthritic mice exhibited a significant capacity for cell migration toward thrombin-cleaved OPN but not toward full-length OPN. Migratory monocytes expressed $\alpha 9$ and $\alpha 4$ integrins. Since cleavage of OPN by thrombin exposes the cryptic epitope recognized by $\alpha 9$ and $\alpha 4$ integrins, we investigated the role of the cryptic epitope SLAYGLR in a murine RA model by using a specific antibody (M5) reacting to SLAYGLR sequence. The M5 antibody could abrogate monocyte migration toward the thrombin-cleaved form of OPN. Importantly, M5 antibody could inhibit the proliferation of synovium, bone erosion, and inflammatory cell infiltration in arthritic joints. Thus, we demonstrated that a cryptic epitope, the SLAYGLR sequence of murine OPN, is critically involved in the pathogenesis of a murine model of RA.
\end{abstract}

J. Clin. Invest. 112:181-188 (2003). doi:10.1172/JCI200317778.

\section{Introduction}

Rheumatoid arthritis (RA) is a chronic inflammatory disease characterized by synovial inflammation and hyperplasia leading to progressive cartilage and bone destruction in which various inflammatory cytokines, such as TNF- $\alpha$ and IL-1, are involved (1). Anti-TNF- $\alpha$ antibody and IL-1 receptor antagonist are effective in the prevention of both inflammation and joint erosion in early active RA; however, they are unable to completely stop the progression of joint destruction in patients with RA, indicating that the pathological process of RA is complex and that other factors are critically involved (2-5). Osteopontin (OPN) has been suggested as potential mediator of the promotion of joint destruction in patients with RA through the $\alpha v \beta 3$ and $\alpha v \beta 5$ integrins expressed on osteoclasts and chondrocytes $(6-11)$. OPN is an extracellular matrix protein

Received for publication January 7, 2003, and accepted in revised form April 22, 2003.

Address correspondence to: Nobuchika Yamamoto, Exploratory Research Laboratories, Fujisawa Pharmaceutical Co., Tokodai,

5-2-3, Tsukuba, Ibaraki, 300-2698, Japan.

Phone: 81-029-847-8611; Fax: 81-029-847-1536;

E-mail: nobuchika_yamamoto@po.fujisawa.co.jp.

Conflict of interest: The authors have declared that no conflict of interest exists.

Nonstandard abbreviations used: osteopontin (OPN);

rheumatoid arthritis (RA); parathyroid hormone (PTH);

macrophage colony-stimulating factor (M-CSF);

tartrate-resistant acid phosphatase (TRAP). containing an Arg-Gly-Asp (RGD) sequence and upregulated in activated $T$ cells, macrophages, invading synoviocytes, and articular chondrocytes associated with inflammation and tissue repair (12-14). It has diverse functions, including cell adhesion, chemotaxis, and immunomodulation through interaction with integrins such as $\alpha v \beta 3$ and $\alpha v \beta 5$ (15). Recent studies show that proteolytic modification of OPN by thrombin cleavage reveals cryptic binding sites for $\alpha 9 \beta 1$ and $\alpha 4 \beta 1$ integrins, preferentially expressed by neutrophils and by monocytes and lymphocytes, respectively $(16-18)$. The newly exposed binding site within OPN, SVVYGLR, promotes adhesion and migration of leukocytes and neutrophils through these alternative sites in an RGD-independent manner (19). In addition, it has been shown that not only macrophages and lymphocytes but also neutrophils play an essential role in the pathogenesis of RA (20-23). Moreover, in RA synovial fluids, reduced levels of coagulation factors with concomitantly increased concentrations of thrombin activity and thrombin/antithrombin complexes have been found, reflecting activation of the coagulation cascade (24-26). Thus, it is conceivable that the thrombincleaved form of OPN plays an important role in the development of arthritis.

To investigate whether the cryptic epitope of OPN generated by thrombin digestion is critically involved in the pathogenesis of RA, we previously generated the specific $\mathrm{mAb} 2 \mathrm{~K} 1$ reacting to the SVVYGLR 
sequence of human OPN. We found that $2 \mathrm{~K} 1$ monoclonal antibody could abrogate the interaction between human OPN and $\alpha 9 \beta 1$ integrin (27). Although the classical $\alpha v$ integrin binding sequence GRGDS and thrombin cleavage sequence YGLRS are well conserved in various species, the cryptic epitope - SVVYGLR sequence in human OPN - is replaced by SLAYGLR in rat and mouse OPN $(16,28)$. One possible approach to test the pivotal role of this cryptic epitope in a murine model of RA is to raise the specific antibody recognizing SLAYGLR. Thus, we have obtained the specific antisera (M5 Ab) reacting to SLAYGLR peptide. We examined the effect of M5 $\mathrm{Ab}$ in the murine arthritis model induced by a mixture of four anti-type II collagen monoclonal antibodies and LPS. We found that M5 Ab reacting with the SLAYGLR sequence exposed by thrombin cleavage of murine OPN significantly suppressed the development of arthritis in mice.

\section{Methods}

M5 antibody. A purified IgG fraction of rabbit sera immunized with synthetic peptide (VDVPNGRGDSLAYGLRS), referred to as M5 Ab, was used in this study.

BIAcore analysis. All experiments were performed at $25^{\circ} \mathrm{C}$ with a flow rate of $10 \mu \mathrm{l}$ per minute using a BIAcore 2000 (BIAcore, Tokyo, Japan). Biotinylated ligands (SLAYGLR, GRGDS, or GRGDSLAYGLR peptide) were bound on the sensor chip SA (BIAcore), and then M5 Ab at $5 \mu \mathrm{g} / \mathrm{ml}$ in HBS buffer ( $10 \mathrm{mM}$ HEPES, $150 \mathrm{mM} \mathrm{NaCl}, 3 \mathrm{mM}$ EDTA, $0.005 \%$ P20 surfectant [pH 7.4]) was injected. The surface plasmon resonance intensity was monitored.

In vitro migration assay. In vitro splenic monocyte migration was evaluated by using a 48-well microchemotaxis chamber (NeuroProbe, Gaithersburg, Maryland, USA) with polycarbonate filter (pore size, 5 $\mu \mathrm{m})$. Recombinant murine OPN (Genzyme-Techne, Minneapolis, Minnesota, USA) was digested by thrombin (Sigma-Aldrich, St. Louis, Missouri, USA) at $5 \mu \mathrm{g}$ of OPN per $2.0 \mathrm{U}$ of enzyme at $37^{\circ} \mathrm{C}$ for 1 hour and used as a chemoattractant. The thrombin-cleaved OPN was incubated with the following antibodies: M5 $\mathrm{Ab}$, anti-OPN N-terminal antisera (LB4225; LSL/ Cosmo Bio, Tokyo, Japan), anti-OPN polyclonal Ab (number 42808; Genzyme-Techne), anti- $\beta 3$ integrin (2C9; BD PharMingen, San Diego, California, USA), and anti-CD44 (IM7; BD PharMingen) at $37^{\circ} \mathrm{C}$ for 15 minutes. For migration assay, splenic monocytes were prepared by collecting adherent cells on a plastic dish, and a majority of the cells expressed CD11c. After incubation at $37^{\circ} \mathrm{C}$ for 2 hours, the migrated cell numbers were quantitated by cell counts of 100 fields by using 100 ocular grids $(\times 40)$.

Organ culture of neonatal mouse calvaria. The organ culture of neonatal mouse calvaria has been previously described in detail (29). Calvarias (frontal and parietal bones) were removed from 1- or 2-day-old C57BL/ 6 mice and cultured for 7 days in $2 \mathrm{ml}$ of
DMEM supplemented with $10 \%$ fetal bovine serum and penicillin $(100 \mathrm{U} / \mathrm{ml})$ with either human parathyroid hormone (PTH) or IL-1 $\alpha$ (Genzyme-Techne). The effects of M5 Ab $(200 \mu \mathrm{g} / \mathrm{ml})$ or anti- $\beta 3$ integrin $\mathrm{Ab}(200 \mu \mathrm{g} / \mathrm{ml})$ were evaluated. Normal rabbit and rat IgG (R\&D Systems, Poquoson, Virginia, USA) were used as isotype control antibodies. The calcium concentration in media was measured using a commercial kit (Wako Pure Chemical Industries Ltd., Osaka, Japan).

Osteoclast formation assay. Bone marrow cells obtained from 8-week-old female DBA/1 mice (Charles River, Shizuoka, Japan) were suspended in $\alpha$-MEM supplemented with $5 \%$ mouse serum and plated in flat-bottom 96 -well plates $\left(2 \times 10^{5}\right.$ cells per well). The cells were incubated with $100 \mathrm{ng} / \mathrm{ml}$ murine M-CSF (Immuno-Biological Lab) and 30 $\mathrm{ng} / \mathrm{ml}$ murine RANKL (Immuno-Biological Lab) in the presence or absence of M5 Ab for 7 days; the culture media was replaced every 3 days. Mature osteoclasts were visualized by a TRAP staining kit (Hokudo, Hokkaido, Japan) and counted microscopically.

Induction and clinical assessment of arthritis in mice. Arthritis was induced by using an arthritogenic mAb cocktail kit (Immuno-Biological Lab). Briefly, 7-weekold female C57BL/6 mice (Charles River, Shizuoka, Japan) were injected intravenously with a mixture of four anti-type II collagen mAbs ( $2 \mathrm{mg}$ each) on day -4 followed by intraperitoneal injection with $100 \mu \mathrm{g}$ of LPS (0111:B4) on day 0 (30). M5 Ab was administered intravenously at doses of 40,150 , and $400 \mu \mathrm{g}$ per mouse on days 0 and 3 . The arthritic group was intravenously administered $400 \mu \mathrm{g}$ of rabbit IgG. The clinical severity of arthritis was graded up to 6 days after LPS administration in each of the four paws on a 1-3 scale as described previously (31). The disease severity was recorded for each limb as follows: 0 , normal; 1 , slight swelling and/or redness in one joint; 2 , moderate swelling in the entire paw; and 3, deformity and/or ankylosis.

Induction of arthritis by type II collagen was performed by the following methods. Male DBA $/ 1$ mice ( 7 weeks old) were immunized at the tail base with $200 \mu \mathrm{g}$ of native bovine type II collagen (Cosmo Bio, Tokyo, Japan) emulsified in CFA containing Mycobacterium tuberculosis strain H37Rv (Wako Pure Chemical Industries Ltd.) and boosted with the same preparations of collagen plus CFA 3 weeks later. Then, the progress of the arthritis was observed for 2 weeks. M5 Ab was administered intravenously at $400 \mu \mathrm{g}$ per mouse twice a week after the boost (a total of four times).

Histology. Histological assessment of the arthritic joint of four paws (ankles and interphalanges) on day 14 were assessed by staining with fast green/safranin O or hematoxylin and eosin. The degrees of synovial proliferation, leukocyte infiltration, and cartilage degeneration were graded as follows: 0 , normal, 1 , mild proliferation of synovium and minor leukocyte infiltration into synovium or minor erosion of the 
cartilage; 2 , invasion of synovium into the joint space and moderate leukocyte infiltration or mild pannus erosion of the cartilage; and 3, extensive leukocyte infiltration into the joint space, fibrous ankylosis of the joints, and peripheral and subchondral cartilage erosion.

Flow cytometric analysis. Alteration of expression of integrins on splenocytes caused by onset of arthritis was analyzed by FACScan (Becton Dickinson, Franklin Lakes, New Jersey, USA). Cells were stained with anti$\alpha 4$ integrin, anti- $\beta 3$ integrin, and anti-CD44.

$R T-P C R$ analysis. Total RNA from spleens and synovial tissues from ankles were extracted by commercial kit (Promega, Madison, Wisconsin, USA). In each group, joint extracts of three mice were pooled. RT-PCR was performed using specific primers. Primers for IL- $1 \beta$, TNF- $\alpha$, IL-6, IL-10, IFN- $\gamma$, and GAPDH were obtained from TOYOBO (Osaka, Japan). Primer sequences for OPN, $\alpha 9$ integrin, and $\alpha 4$ integrin were as follows: OPN (5'-ATGAGATTGGCAGTGATTTGCTT-3' and 5'TTAGTTGACCTCAGAAGATGCACTCT-3'), $\alpha 9$ integrin (5'-AAAGGCTGCAGCTGTCCCACATGGACGAAG-3' and $5^{\prime}$-TTTAGAGAGATATTCTTCACAGCCCCCAAA-3'), and $\alpha 4$ integrin (5'-TGGAAGCTACTTAGGCTACT- $3^{\prime}$ and $5^{\prime}$ TCCCACGACTTCGGTAGTAT- $\left.3^{\prime}\right)$. Cycling conditions were $94^{\circ} \mathrm{C}$ for 30 seconds, $55^{\circ} \mathrm{C}$ for 30 seconds, and $72^{\circ} \mathrm{C}$ for 1 minute.

Statistical analysis. Statistical evaluation was performed based on Dunnet's multiple comparison test, or the Wilcoxon/Kruskal-Wallis rank-sum test for unpaired variables (two-tailed) was used to compare the differences between groups. $P$ values less than 0.05 were considered significant. a

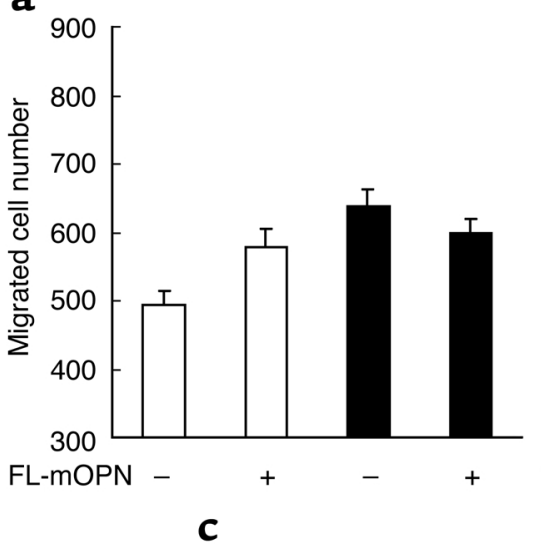

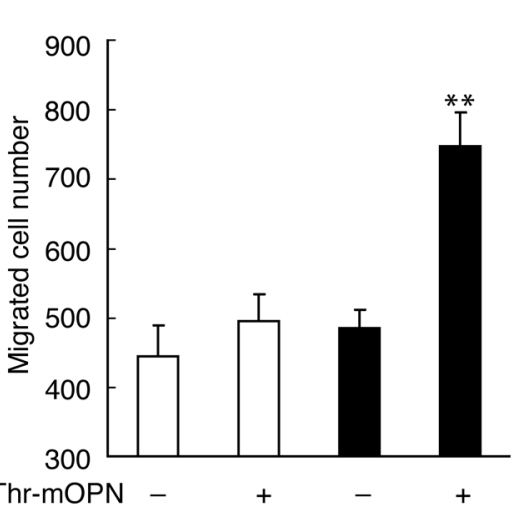

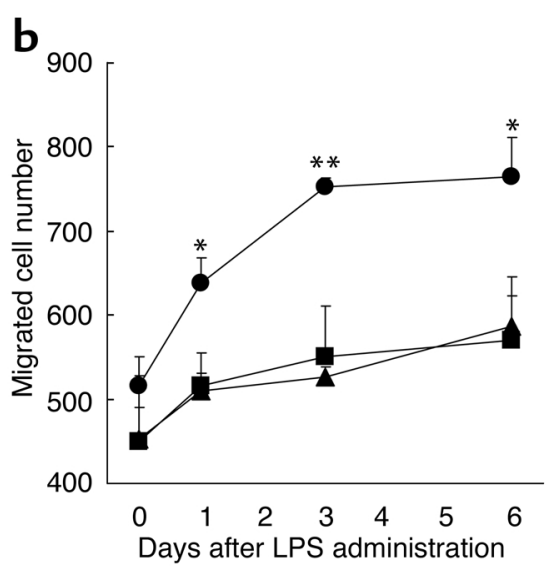

$\alpha 9$ Integrin

(343 bp)

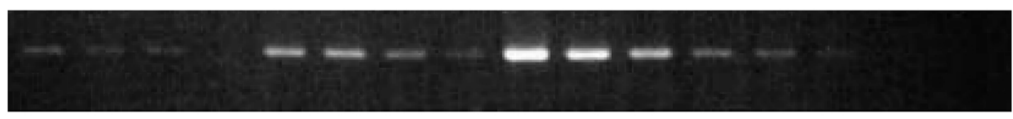

Template cDNA

GAPDH

(453 bp)

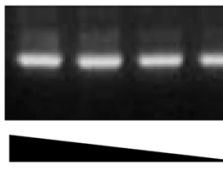

Day 0

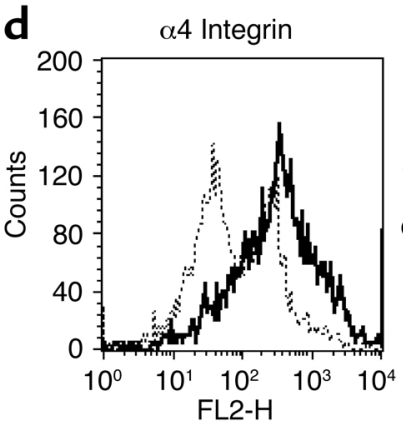

Day 1

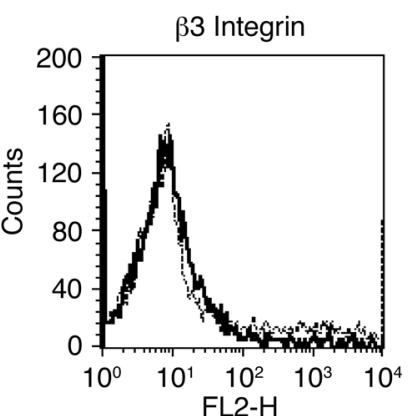

Day 3

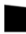

Day 6

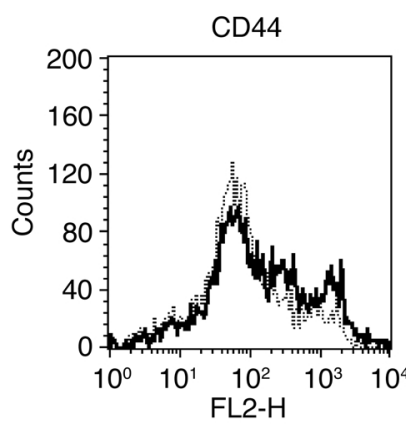

Figure 1

Monocyte migration in response to thrombin-cleaved OPN. (a) Splenic monocytes from normal (white bars) and arthritic (black bars) mice on day 6 of migration in response to full-length murine OPN (FL-mOPN) and thrombin-cleaved murine OPN (Thr-mOPN) at 10 $\mu \mathrm{g} / \mathrm{ml}$. (b) The time course of the migration response toward medium (square), full-length (triangle), and thrombin-cleaved OPN (circle) was evaluated. The results are expressed as mean numbers \pm SEM of migrated cells. ${ }^{*} P<0.05$ and ${ }^{*} P<0.01$. (c) Expression of $\alpha 9$ integrin mRNA levels in spleen of arthritic mice on days $0,1,3$, and 6 after LPS administration was analyzed using RT-PCR and GAPDH as a housekeeping gene. Template cDNA was diluted sequentially (left to right) and amplified by PCR. (d) Expressions of $\alpha 4$ and $\beta 3$ integrins and CD44 on splenocytes obtained from normal (dotted line) and arthritic (solid line) mice were analyzed by FACS. Histograms represent the mean fluorescence intensity. Controls are from isotype-matched irrelevant antibodies. 


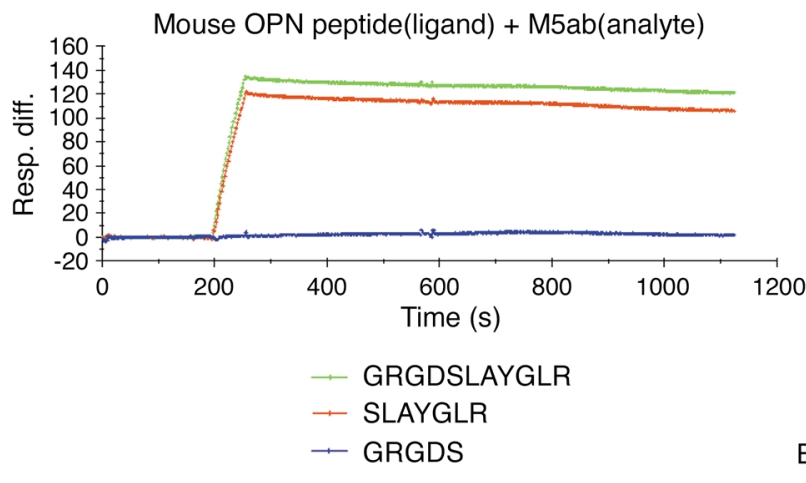

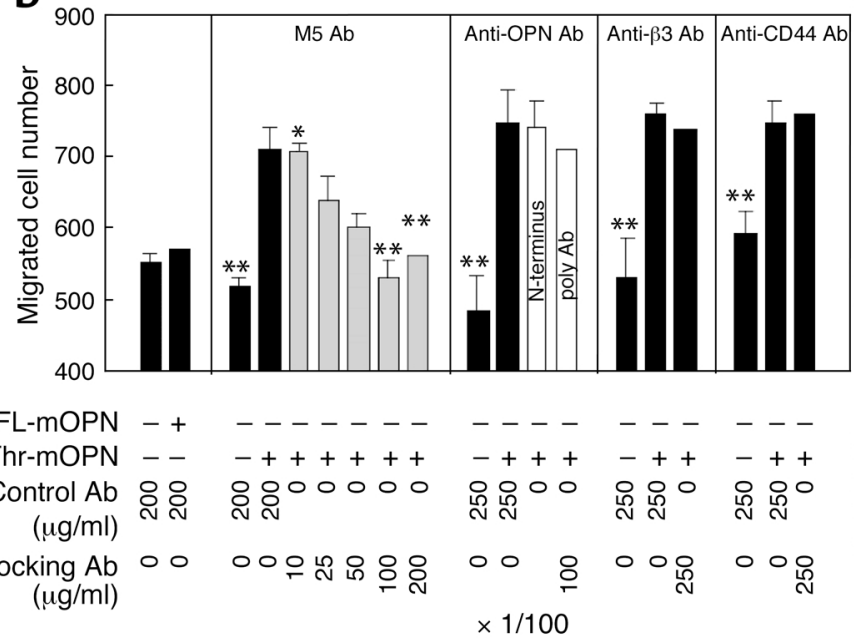

\section{Figure 2}

M5 Ab inhibited migration of splenic monocytes from arthritic mice. (a) Specificity of M5 Ab against OPN peptides was examined by analysis using BIAcore analysis of M5 Ab. Vertical and horizontal axes indicate surface plasmon resonance intensity (units) and flow time of HBS buffer, respectively. M5 Ab was injected at 200 seconds over the surface of the chip and then washed with HBS buffer. The binding of M5 Ab to GRGDSLAYGLR peptide (green), SLAYGLR peptide (red), and GRGDS peptide (blue) is shown. (b) Inhibition by M5 Ab of cell migration toward thrombin-cleaved OPN. M5 Ab, antipolyclonal OPN Abs, anti- $\beta 3$ integrin Ab, anti-CD44 Ab, and isotype-matched control antibodies were added at the indicated concentrations. Anti-N-terminal OPN was added at a 1:100 dilution to wells. The results are expressed as mean numbers \pm SEM of migrated cells. ${ }^{*} P<0.05$ and ${ }^{*} P<0.01$. Resp. diff., response difference.

\section{Results}

Augmented migration of monocytes from arthritic mice toward thrombin-cleaved OPN paralleled with the $\alpha 9$ and $\alpha 4$ integrin expression. Splenic monocytes of normal mice did not show a significant level of migration toward full-length and thrombin-cleaved forms of murine OPN. In contrast, monocytes from arthritic mice exhibited significant migratory activity toward the thrombin-cleaved form but not the full-length murine OPN (Figure 1a). The migratory activity was evident at day 1 and reached a plateau at day 3 (Figure 1b). To examine whether the augmented migratory activity of monocytes is related to the arthritis but not the simple consequence of LPS injection, we obtained monocytes of mice treated with LPS alone and found that monocytes did not migrate toward OPN (data not shown). We next examined the expression of OPN receptors on splenic monocytes from control and arthritic mice. The expression of $\alpha 9$ integrin began to increase on day 1 , reached peak on day 3 , and declined on day 6 (Figure 1c). The expression of $\alpha 4$ integrin also increased on day 3 . However, the expressions of $\beta 3$ integrin and CD44 did not change on day 3 (Figure 1d). Thus, the increased expression of $\alpha 9$ and $\alpha 4$ integrin paralleled with the increase of migratory activity of splenic monocytes toward thrombincleaved murine OPN. The thrombin-cleaved form of human OPN exposed a cryptic epitope, SVVYGLR, that contained both $\alpha 9$ and $\alpha 4$ integrin binding sites (16, 32). To assess the importance of SLAYGLR sequence within murine OPN, which corresponds to the SVVLYGR sequence in human OPN, we prepared M5 Ab, which specifically recognizes SLAYGLR. We first examined the specificity of M5 Ab by BIAcore. We have found that M5 Ab could equally bind to the immunizing peptide GRGDSLAYGLR and SLAYGLR but failed to bind to the $\alpha v \beta 3$ integrin binding motif, GRGDS (Figure 2a). We have examined whether M5 Ab crossreacts with molecule(s) other than the cryptic epitope of OPN. VCAM-1 is one of the ligands of $\alpha 4$ and $\alpha 9$ integrin and is known to participate in leukocyte migration (19). We found that M5 Ab did not bind to VCAM-1 (data not shown). The splenic monocytes obtained from arthritic mice could migrate toward the thrombin-cleaved form of murine OPN but not the full-length form of murine OPN (Figure 2b). The monocyte migration toward the thrombin-cleaved form of OPN was not inhibited by anti- $\beta 3$ integrin $\mathrm{mAb}$, anti-CD44 Ab, and control polyclonal antibodies. In contrast, M5 Ab could almost completely inhibit the cell migration, indicating that the SLAYGLR motif, but not the GRGDS motif, is critically involved in the cell migration against thrombin-cleaved OPN.

Protection against antibody-induced arthritis by M5 Ab correlates with inbibition of degeneration and inflammatory reaction, normal joint morphology, and improvement of general condition. We demonstrated that M5 Ab possessed a prophylactic effect on arthritis. Treatment with M5 Ab on days 0 and 3 led to the delay of clinical onset of arthritis (Figure 3b). However, by day 5 all mice had developed arthritis. Importantly, the severity of arthritis was significantly attenuated by M5 Ab treatment (Figure 3a). The gross appearance of arthritic joint treated with M5 $\mathrm{Ab}$ was similar to that of normal mice (Figure $3 \mathrm{c}$ ). The systemic inflammatory responses in arthritic mice were reflected by the severe reduction in food intake and the loss of body weight on day 6 (Figure 3, d and e). Upon 
M5 Ab treatment, food intake was significantly improved (approximately $85 \%$ of control mice) and loss of body weight was inhibited. Thus, the general condition in arthritic mice was significantly improved after M5 Ab treatment. Moreover, therapeutic administration of M5 Ab after the onset of clinical symptom on day 3 markedly reduced the clinical score of arthritis (Figure $3 \mathrm{f}$ ). We also examined whether M5 Ab could inhibit the occurrence of arthritis in a murine model of type II collagen-induced arthritis. We found that $80 \%$ of mice developed arthritis by day 7 with type II collagen, whereas only $20 \%$ of mice developed arthritis after M5 Ab treatment (data not shown). Next, we evaluated the effect of M5 Ab on joint histology on day 14 after LPS injection (Figure 4, a-i). The significant suppression of synovial hyperplasia (Figure $4 \mathrm{f})(P=0.0035)$, leukocyte infiltration (mainly polymorphonuclear leukocytes) (Figure 4f) $(P=0.0015)$, and erosion of joint cartilage (Figure $4 c)(P=0.0123)$ was evident in M5 Abtreated mice (Figure $4, \mathrm{j}-1)$.

M5 Ab inbibited osteoclast-mediated bone resorption and the expression of cytokine in arthritic joint. We further clarified the molecular mechanism of M5 Ab action on
PTH-induced and IL- $1 \alpha$-induced bone resorption in vitro. PTH as well as IL-1 induced release of calcium into medium (Figure 5, a and b). M5 Ab completely inhibited the calcium release. PTH-induced bone resorption was only partially inhibited by the anti- $\beta 3$ integrin $\mathrm{Ab}$ (Figure $5 \mathrm{c}$ ), indicating that $\alpha \mathrm{v}$ integrin is only partially responsible for PTH- and IL- $1 \alpha$-induced bone resorption. Since PTH-induced bone resorption involved receptor activator of the NF- $\mathrm{KB}$ ligand (RANKL)/RANK pathway (33-35), we investigated whether the interaction of SLAYGLR and its receptor is located downstream of RANKL and murine macrophage colony-stimulating factor (M-CSF). Thus, bone marrow cell-derived progenitor was cultured in the presence of M-CSF and RANKL. M5 Ab could inhibit the number of tartrate-resistant acid phosphatase-positive (TRAP-positive) cells (Figure 5d). We next tested in vivo interference of M5 Ab on proinflammatory cytokine expression in arthritic joints. Expressions of IL-1 $\beta$, TNF- $\alpha$, IL- 6 , and IL-10 were hardly present in joint tissues of normal mice but were clearly detected in arthritic joints (Figure 5e). M5 Abtreated mice showed a marked decrease in expression $\mathbf{a}$

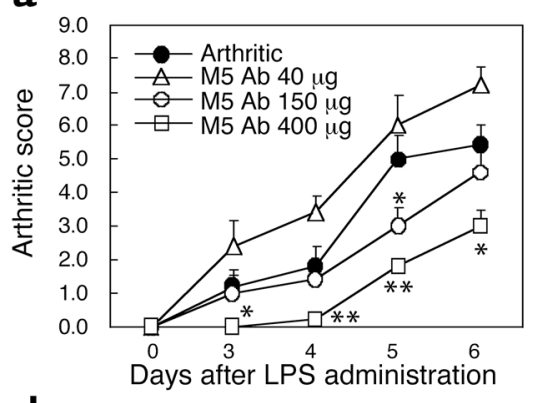

d

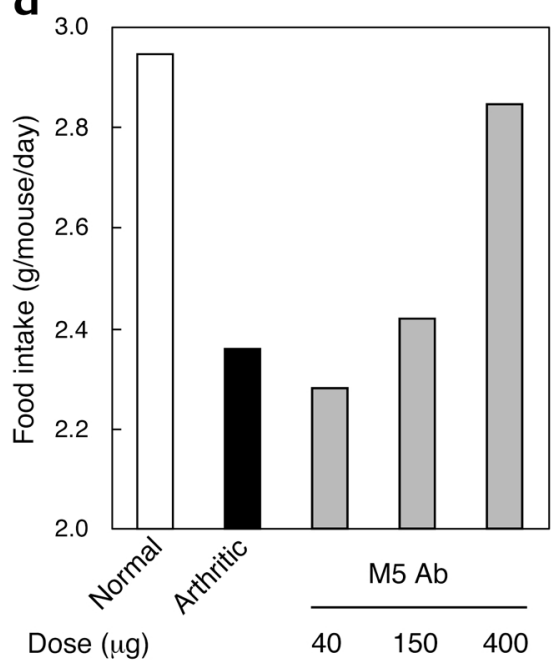

b

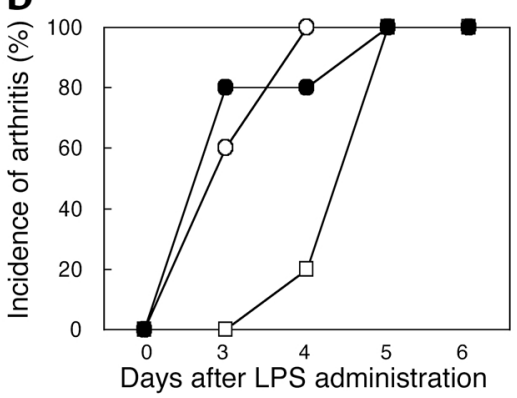

e

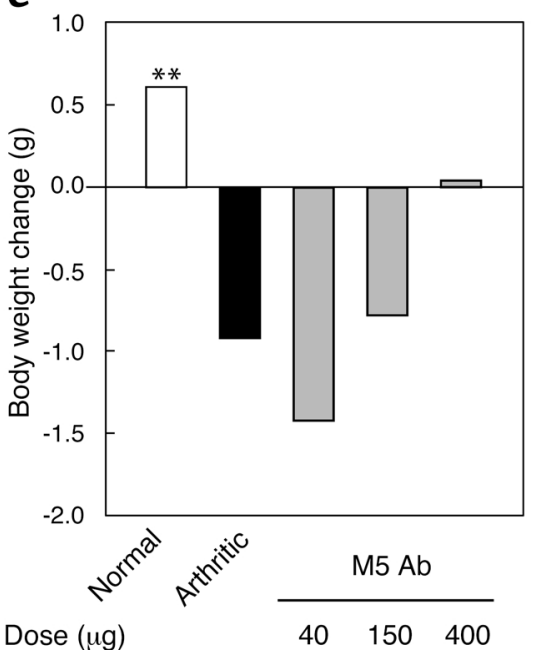

c

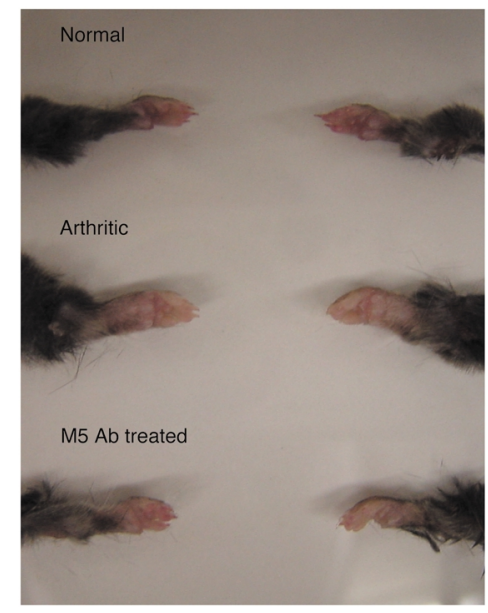

f

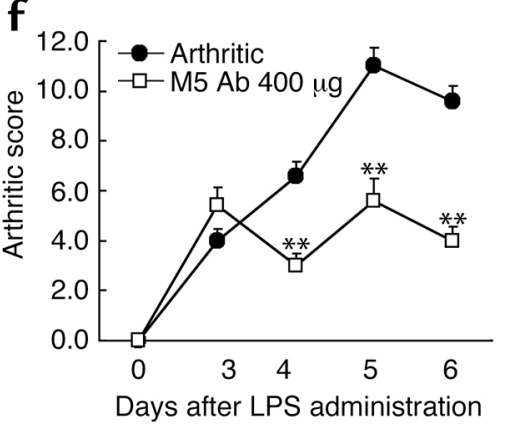

Figure 3

Prophylactic and therapeutic treatment with M5 Ab ameliorated symptoms of arthritis. M5 Ab was administered intravenously at doses of 40,150 , and $400 \mu \mathrm{g}$ per mouse before the onset of clinical symptoms on days 0 and 3 . The arthritic group was intravenously administered $400 \mu \mathrm{g}$ of rabbit IgG. Arthritic score (a), incidence of arthritis (b), food intake (d), and body weight (e) were monitored. Representative gross appearances of the forepaw are shown (c). Arthritic mice were therapeutically treated with M5 Ab after the onset of symptoms on day 3 , and arthritic scores were monitored $(\mathbf{f})$. Each point represents the mean score \pm SEM of five mice. ${ }^{*} P<0.05$ and ${ }^{*} P<0.01$ for comparison by Mann-Whitney $U$ test with arthritic mice; ${ }^{\#} P<0.01$ for comparison by Dunnet's multiple-comparison test with arthritic mice. 


\section{Figure 4}

Histology of arthritic joints after M5 Ab treatment. Mice receiving prophylactic treatment of $400 \mu \mathrm{g}$ per mouse (intravenous injection) of M5 Ab (Figure 3) $(n=5)$ were analyzed. The sections of ankle joints in fore and hind paws were stained with either safranin $\mathrm{O}(\mathbf{a}-\mathbf{c})$ or hematoxylin and eosin (d-i), and representative histological images of hind paws are shown: normal ( $\mathbf{a}$ and $\mathbf{d}$ ), arthritic ( $\mathbf{b}$ and $\mathbf{e}$ ) and M5 Ab treated ( $c$ and $\mathbf{f}$ ). The image of leukocytes from an arthritic joint was magnified ( $\mathbf{g}-\mathbf{i}$ ). Hyperplasia of synovium (j), leukocyte infiltration (k), and cartilage degeneration (I) were quantified. Histological scores are expressed as means \pm SEM of four paws of five mice. ${ }^{*} P<0.05$ and ${ }^{*} P<0.01$ for comparison by Mann-Whitney $U$ test with arthritic mice.
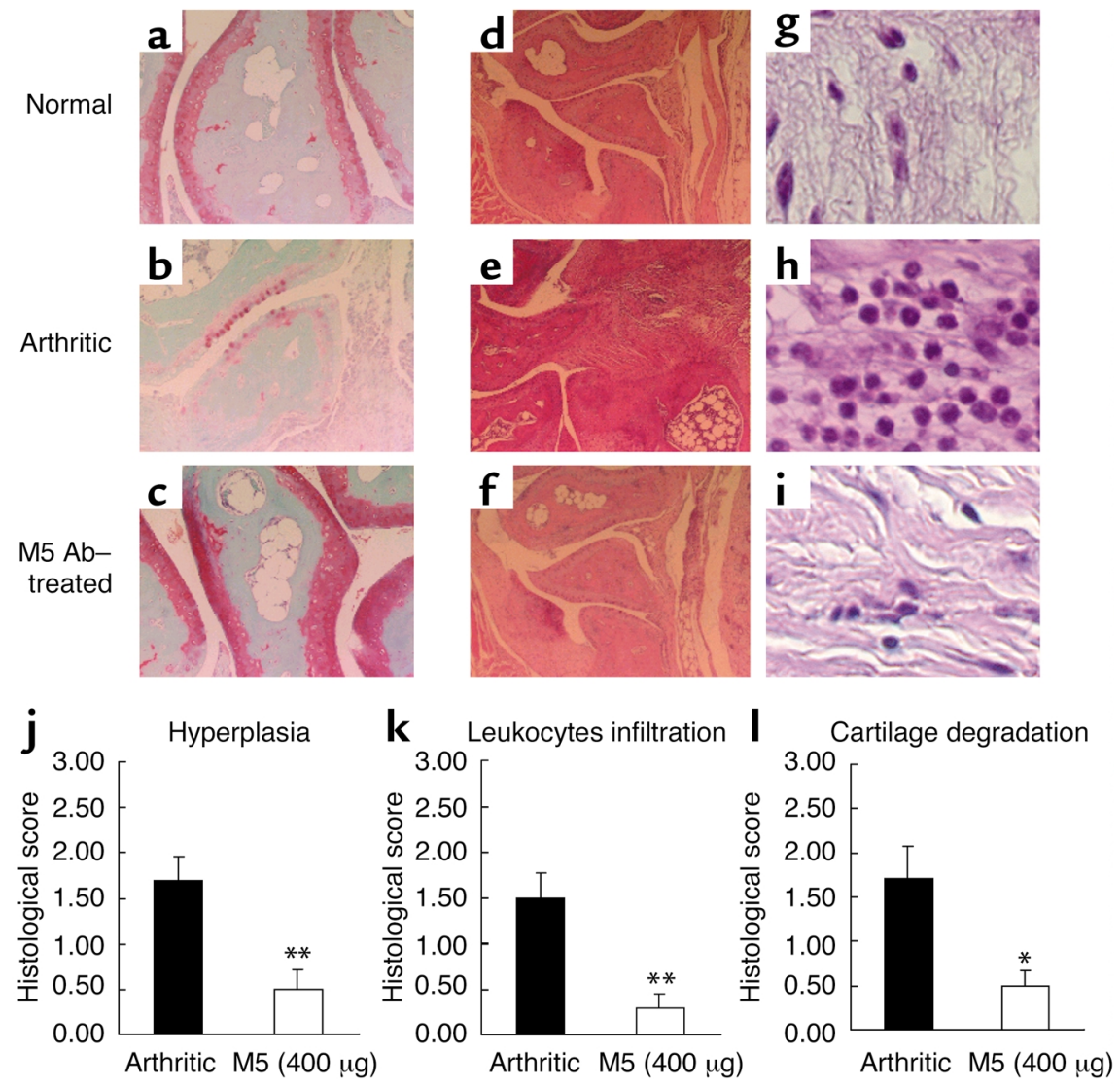

of those cytokines in joint extracts. We also found the expression of $\alpha 4$ and $\alpha 9$ integrins in arthritic joins, and the expression was suppressed by M5 Ab treatment. In contrast, the expression of IFN- $\gamma$ seemed to be the same in three variants.

\section{Discussion}

RA is a chronic autoimmune inflammatory disease characterized by progressive destruction of bone and cartilage of joint. Various inflammatory cytokines including TNF- $\alpha$, IL-1, and IL- 6 stimulate the proliferation of synovial cells, which resulted in the formation of pannus, and activated osteoclasts within pannus degrade bone tissues (36). The attachment of activated osteoclasts to the bone surface through integrin receptors is a critical step in bone resorption by osteoclasts (37).

Recently, the molecular mechanism that involves in the bone resorption by osteoclasts has been substantially clarified. PTH and possibly IL-1 induce the expression and secretion of RANKL and M-CSF from osteoblasts, and those factors bind to respective receptors on osteoclasts, thus regulating the differentiation and activation of osteoclasts $(33-35,38)$. PTH-induced bone resorption and bone resorption induced by RANKL and M-CSF do not occur in the absence of OPN (7). PTH-induced increase in TRAP-positive osteoclasts from bone marrow progenitor cells was deficient in OPN null mice (7). Thus, the differentiation and activation of osteoclasts are OPN dependent, and OPN is located downstream of the RANKL/RANK pathway. Bone resorption by osteoclasts is regulated by the interaction of $\alpha v \beta 3$ integrin on osteoclasts and OPN (39). Indeed, we recently found that OPN and its receptor $\alpha v \beta 3$ integrin were coexpressed by activated osteoclasts at the site of bone erosion (10).

However, anti- $\beta 3$ integrin $\mathrm{Ab}$, which interferes with the binding between $\alpha v \beta 3$ integrin and OPN, could only partially inhibit bone resorption induced by PTH. Alternatively, it is conceivable that the cryptic OPN epitope SLAYGLR bound to $\alpha 9$ or $\alpha 4$ integrin receptor on osteoclasts and stimulated differentiation and activation of osteoclasts. This hypothesis is consistent with our findings: (a) osteoclasts derived from bone marrow cells stimulated with RANKL and M-CSF expressed $\alpha 9$ integrin mRNA but not $\alpha 4$ integrin (data not shown), and (b) M5 Ab could significantly inhibit the number of TRAP-positive cells from the progenitor bone marrow cells. In this regard, it is of importance to define whether the cleaved form of OPN is present in IL-1- or PTH-stimulated calvaria in the future. OPN plays a critical role in several distinct steps during the course of RA. In addition to the activation and differentiation of osteoclasts by OPN, the inflammatory infiltrates within joint tissue were also significantly suppressed in the absence of OPN (11). This is consistent with previous findings that OPN is chemotactic for various inflammatory cells such as macrophages, neutrophils, and lymphocytes $(18,19)$. It is likely that the binding of 

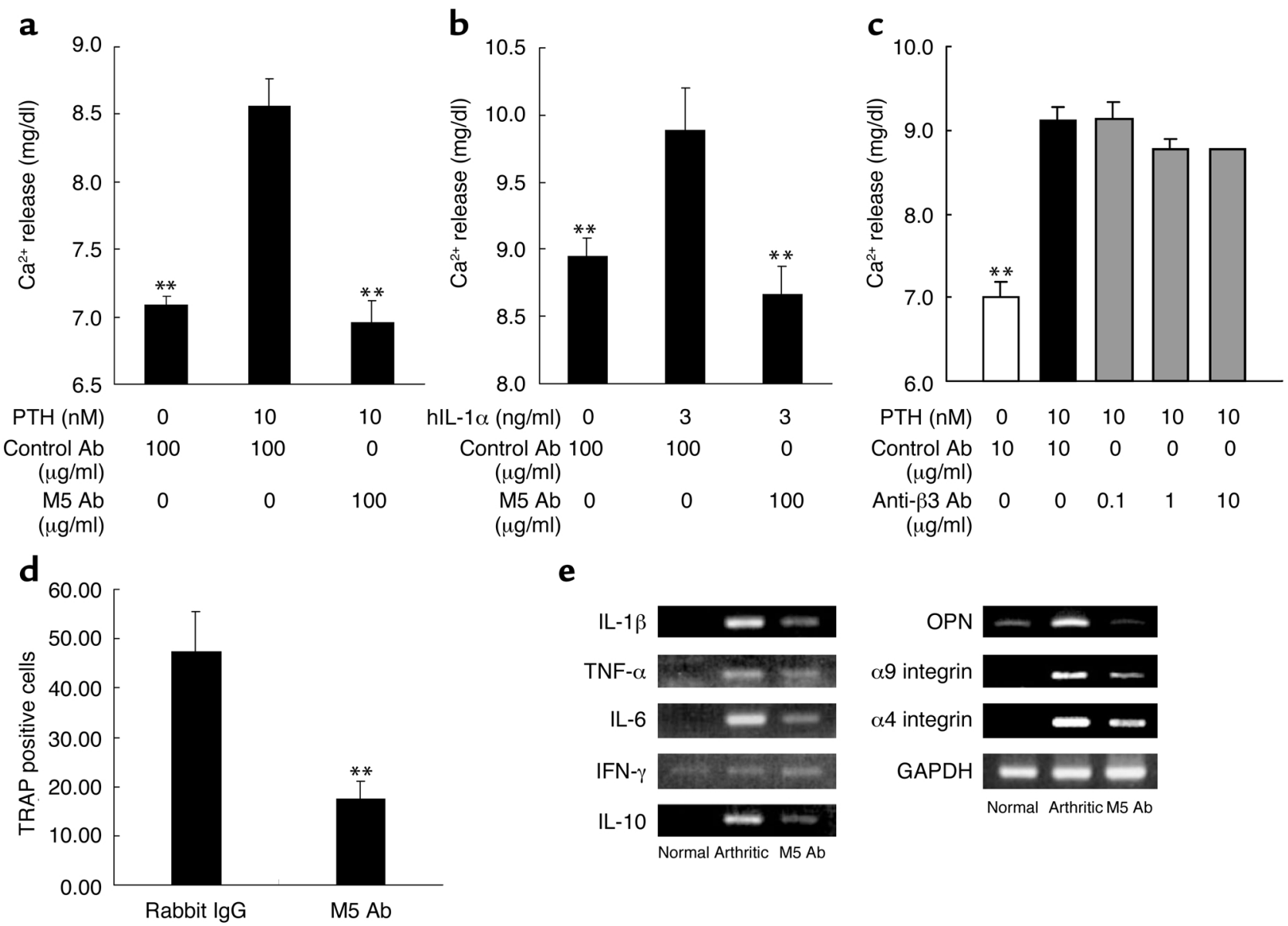

e
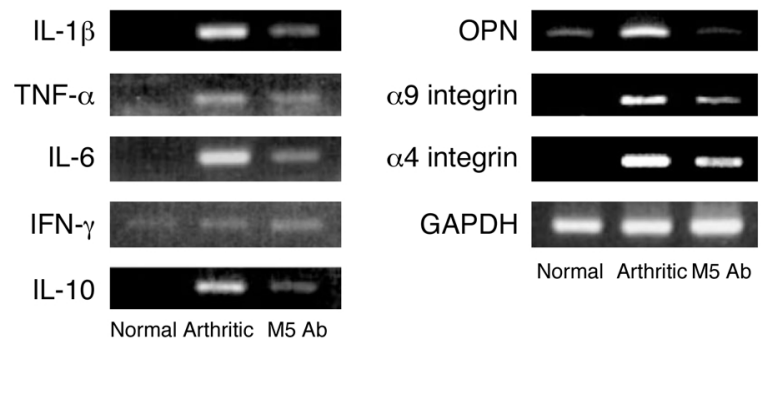

Figure 5

M5 Ab prevented osteoclast-mediated bone resorption and osteoclast formation in vitro. (a and b) PTH or IL-1 $\alpha$ induced calcium releases. PTH and IL- 1 induced calcium release from bone in murine neonatal calvaria culture in the presence of M5 Ab (a and $\mathbf{b})$ or anti- $\beta 3$ integrin $\mathrm{Ab}(\mathbf{c})$. Results are expressed as means $\pm \mathrm{SEM}$. ${ }^{*} P<0.01$ for comparison by Dunnet's multiple-comparison test with isotype control lgG plus PTH or IL-1 $\alpha$. (d) Mouse bone marrow cells were cultured for 7 days in the presence of M-CSF and RANKL in the presence of control antibody or M5 Ab $(200 \mu \mathrm{g} / \mathrm{ml})$, and TRAP-positive mature osteoclasts numbers were counted and expressed as mean numbers \pm SEM of cells. (e) Arthritic mice prophylactically treated with M5 Ab on day 0 and 3 in Figure 3 were used. Expressions of proinflammatory cytokines, OPN, and integrin mRNA in joint extracts were analyzed using RT-PCR and GAPDH as a housekeeping gene. These are representative data from three separate experiments.

OPN to inflammatory cells is mediated by $\alpha v \beta 3$ integrin. However, it was shown that $\alpha v \beta 3$ integrin antagonists such as SB273005 and cyclic RGD peptide could ameliorate joint destruction without blocking the inflammatory response in animal models of RA (40, 41 ), suggesting that the interaction of OPN with receptors other than $\alpha v \beta 3$ integrin may participate in the inflammatory response.

The critical issue to be asked here is which portion of OPN and what receptor are involved in the inflammatory responses in RA. It is known that the coagulation activity is enhanced in patients with RA and that the levels of complex of thrombin/thrombin inhibitor are higher than in normal subjects (24). We recently found that the thrombin-cleaved form of OPN is elevated in synovial fluid of RA (42). In this study, we found the expression of $\alpha 9$ and $\alpha 4$ integrin in arthritic joints and discovered that M5 Ab specifically recognizing SLAYGLR could significantly inhibit the infiltration of inflammatory cells, indicating the critical involvement of SLAYGLR and its receptor in inflammatory cell responses. Finally, cytokines are critically involved in the pathogenesis of RA (2-5). TNF- $\alpha$ and IL-1 were known to induce OPN expression $(43,44)$. In arthritic joints, TNF- $\alpha$, IL-1, IL- 6 , and OPN were coexpressed. The abrogation of the binding between SLAYGLR sequence and its receptor by M5 Ab leads to the downregulation of OPN and cytokines, indicating that autocrine and paracrine induction of OPN are members of the cytokine circuit that are involved in the pathogenesis of RA.

It has been known that patients with RA exhibit a condition described as rheumatoid cachexia (45), which is characterized by a loss of body mass. It is accompanied by elevated resting energy expenditure, accelerated whole-body protein catabolism, and excess production of inflammatory cytokines such as IL-1 and TNF- $\alpha$. At present, there is no standard treatment 
available for rheumatoid cachexia. Since M5 Ab improved food intake and loss of body weight, antiOPN Ab could also be useful for rheumatoid cachexia.

In conclusion, the present study strongly suggests the involvement of the internal sequence of OPN, SLAYGLR, in the pathogenesis of RA. OPN is involved in the osteoclast-mediated bone resorption through the RANK/RANKL pathway. OPN is also involved in the inflammatory responses in joint through the recruitment of inflammatory cells and augments the expression of cytokines (IL-1 $\beta$, TNF- $\alpha$, IL- 6 , and OPN) and integrins ( $\alpha 4$ and $\alpha 9$ ), and these events are likely mediated by the cryptic domain of OPN, SLAYGLR.

1. van der Heijde, D.M. 1995. Joint erosions and patients with early rheumatoid arthritis. Br. J. Rheumatol. 34 (Suppl. 2):74-78.

2. Bathon, J.M., et al. 2000. A comparison of etanercept and methotrexate in patients with early rheumatoid arthritis. N. Engl. J. Med. 343:1586-1593.

3. Campion, G.V., Lebsack, M.E., Lookabaugh, J., Gordon, G., and Catalano, M. 1996. Dose-range and dose-frequency study of recombinant human interleukin-1 receptor antagonist in patients with rheumatoid arthritis. The IL-1Ra Arthritis Study Group. Arthritis Rheum. 39:1092-1101.

4. Paget, S.A. 2002. Efficacy of anakinra in bone: comparison to other biologics. Adv. Ther. 19:27-39.

5. Genovese, M.C., et al. 2002. Etanercept versus methotrexate in patients with early rheumatoid arthritis: Two-year radiographic and clinical outcomes. Arthritis Rheum. 46:1443-1450.

6. Petrow, P.K., et al. 2000. Expression of osteopontin messenger RNA and protein in rheumatoid arthritis: effects of osteopontin on the release of collagenase 1 from articular chondrocytes and synovial fibroblasts. Arthritis Rheum. 43:1597-1605.

7. Ihara, H., et al. 2001. Parathyroid hormone-induced bone resorption does not occur in the absence of osteopontin. J. Biol. Chem. 276:13065-13071.

8. Nakamura, I., Tanaka, H., Rodan, G.A., and Duong, L.T. 1998. Echistatin inhibits the migration of murine prefusion osteoclasts and the formation of multinucleated osteoclast-like cells. Endocrinology. 139:5182-5193.

9. Carron, C.P., et al. 2000. Peptidomimetic antagonists of alphavbeta3 inhibit bone resorption by inhibiting osteoclast bone resorptive activity, not osteoclast adhesion to bone. J. Endocrinol. 165:587-598.

10. Ohshima, S., et al. 2002. Expression of osteopontin at sites of bone erosion in a murine experimental arthritis model of collagen-induced arthritis: possible involvement of osteopontin in bone destruction in arthritis. Arthritis Rheum. 46:1094-1101.

11. Yumoto, K., et al. 2002. Osteopontin deficiency protects joints against destruction in anti-type II collagen antibody-induced arthritis in mice. Proc. Natl. Acad. Sci. U. S. A. 99:4556-4561.

12. Singh, R.P., Patarca, R., Schwartz, J., Singh, P., and Cantor, H. 1990. Definition of a specific interaction between the early $T$ lymphocyte activation 1 (Eta-1) protein and murine macrophages in vitro and its effect upon macrophages in vivo. J. Exp. Med. 171:1931-1942.

13. Denhardt, D.T., and Noda, M. 1998. Osteopontin expression and function: role in bone remodeling. J. Cell Biochem. 30-31(Suppl.):92-102.

14. Denhardt, D.T., Giachelli, C.M., and Rittling, S.R. 2001. Role of osteopontin in cellular signaling and toxicant injury. Annu. Rev. Pharmacol. Toxicol. 41:723-749.

15. Sodek, J., Ganss, B., and McKee, M.D. 2000. Osteopontin. Crit. Rev. Oral Biol. Med. 11:279-303.

16. Yokosaki, Y., et al. 1999. The integrin alpha(9)beta(1) binds to a novel recognition sequence (SVVYGLR) in the thrombin-cleaved amino-terminal fragment of osteopontin. J. Biol. Chem. 274:36328-36334.

17. Smith, L.L., and Giachelli, C.M. 1998. Structural requirements for alpha 9 beta 1-mediated adhesion and migration to thrombin-cleaved osteopontin. Exp. Cell Res. 242:351-360.

18. Bayless, K.J., Meininger, G.A., Scholtz, J.M., and Davis, G.E. 1998. Osteopontin is a ligand for the alpha4beta1 integrin. J. Cell Sci. 111:1165-1174.

19. Taooka, Y., Chen, J., Yednock, T., and Sheppard, D. 1999. The integrin alpha9beta1 mediates adhesion to activated endothelial cells and transendothelial neutrophil migration through interaction with vascular cell adhesion molecule-1. J. Cell Biol. 145:413-420.

20. Feldmann, M., Brennan, F.M., and Maini, R.N. 1996. Role of cytokines in rheumatoid arthritis. Annu. Rev. Immunol. 14:397-440.

21. Brennan, F.M., Maini, R.N., and Feldmann, M. 1998. Role of proinflammatory cytokines in rheumatoid arthritis. Springer Semin. Immunopathol. 20:133-147.

22. Wipke, B.T., and Allen, P.M. 2001. Essential role of neutrophils in the initiation and progression of a murine model of rheumatoid arthritis. J. Immunol. 167:1601-1608.

23. Leung, B.P., et al. 2001. A role for IL-8 in neutrophil activation. J. Immunol. 167:2879-2886.

24. Nakano, S., Ikata, T., Kinoshita, I., Kanematsu, J., and Yasuoka, S. 1999. Characteristics of the protease activity in synovial fluid from patients with rheumatoid arthritis and osteoarthritis. Clin. Exp. Rheumatol. 17:161-170.

25. Furmaniak-Kazmierczak, E., et al. 1994. Studies of thrombin-induced proteoglycan release in the degradation of human and bovine cartilage. J. Clin. Invest. 94:472-480.

26. Ohba, T., Takase, Y., Ohhara, M., and Kasukawa, R. 1996. Thrombin in the synovial fluid of patients with rheumatoid arthritis mediates proliferation of synovial fibroblast-like cells by induction of platelet derived growth factor. J. Rheumatol. 23:1505-1511.

27. Kon, S., et al. 2002. Mapping of functional epitopes of osteopontin by monoclonal antibodies raised against defined internal sequences. J. Cell. Biochem. 84:420-432.

28. Chiba, S., et al. 2000. The role of osteopontin in the development of granulomatous lesions in lung. Microbiol. Immunol. 44:319-332.

29. Conaway, H.H., Grigorie, D., and Lerner, U.H. 1996. Stimulation of neonatal mouse calvarial bone resorption by the glucocorticoids hydrocortisone and dexamethasone. J. Bone Miner. Res. 11:1419-1429.

30. Terato, K., et al. 1992. Induction of arthritis with monoclonal antibodies to collagen. J. Immunol. 148:2103-2108.

31. Seki, N., et al. 1998. Type II collagen-induced murine arthritis. I. Induction and perpetuation of arthritis require synergy between humoral and cell-mediated immunity. J. Immunol. 140:1477-1484.

32. Bayless, K.J., and Davis, G.E. 2001. Identification of dual a4b1 integrin binding sites within a 38 amino acid domain in the $\mathrm{N}$-terminal thrombin fragment of human osteopontin. J. Biol. Chem. 276:13483-13489.

33. Miyamoto, T., et al. 2000. An adherent condition is required for formation of multinuclear osteoclasts in the presence of macrophage colonystimulating factor and receptor activator of nuclear factor kappa B ligand. Blood. 96:4335-4343.

34. Kong, Y.Y., et al. 1999. OPGL is a key regulator of osteoclastogenesis, lymphocyte development and lymph-node organogenesis. Nature. 397:315-323.

35. Kong, Y.Y., et al. 1999. Activated T cells regulate bone loss and joint destruction in adjuvant arthritis through osteoprotegerin ligand. Nature. 402:304-309.

36. Gravallese, E.M., et al. 2000. Synovial tissue in rheumatoid arthritis is a source of osteoclast differentiation factor. Arthritis Rheum. 43:250-258.

37. McHugh, K.P., et al. 2000. Mice lacking beta3 integrins are osteosclerotic because of dysfunctional osteoclasts. J. Clin. Invest. 105:433-440.

38. Hofbauer, L.C., et al. 1999. Interleukin-1beta and tumor necrosis factoralpha, but not interleukin-6, stimulate osteoprotegerin ligand gene expression in human osteoblastic cells. Bone. 25:255-259.

39. Hara, H., et al. 2001. Parathyroid hormone-induced bone resorption does not occur in the absence of osteopontin. J. Biol. Chem. 276:13065-13071.

40. Badger, A.M., et al. 2001. Disease-modifying activity of SB 273005, an orally active, nonpeptide alphavbeta3 (vitronectin receptor) antagonist, in rat adjuvant-induced arthritis. Arthritis Rheum. 44:128-137.

41. Storgard, C.M., et al. 1999. Decreased angiogenesis and arthritic disease in rabbits treated with an alphavbeta3 antagonist. J. Clin. Invest. 103:47-54.

42. Ohshima, S., et al. 2002. Enhanced local production of osteopontin in rheumatoid joints. J. Rheumatol. 29:2061-2067.

43. Miyazaki, Y., et al. 1995. Expression of osteopontin in a macrophage cell line and in transgenic mice with pulmonary fibrosis resulting from the lung expression of a tumor necrosis factor-alpha transgene. Ann. N. Y. Acad. Sci. 760:334-341.

44. Guo, H., Cai, C.Q., Schroeder, R.A., and Kuo, P.C. 2001. Osteopontin is a negative feedback regulator of nitric oxide synthesis in murine macrophages. J. Immunol. 166:1079-1086.

45. Walsmith, J., and Roubenoff, R. 2002. Cachexia in rheumatoid arthritis. Int. J. Cardiol. 85:89-99. 